\title{
Going "paperless" in an English National Health Service (NHS) breast cancer screening service: The intriduction of fully digital mammography
}

\author{
Sian Taylor-Phillips ${ }^{1}$, Amy Grove ${ }^{1 *}$, Sharon Hoffmeister ${ }^{2}$, Margot Wheaton ${ }^{2}$, Sarah Coult ${ }^{2}$, \\ Joanne Essex ${ }^{2}$, Janice Hackney ${ }^{2}$, Sandra Di Cioccio ${ }^{2}$, Aileen Clarke ${ }^{1}$ \\ ${ }^{1}$ Warwick Medical School, Division of Health Sciences, The University of Warwick, Coventry, UK; \\ *Corresponding Author: A.L.Grove@Warwick.ac.uk \\ ${ }^{2}$ Warwickshire, Solihull \& Coventry Breast Screening Unit, University Hospitals Coventry and Warwickshire NHS Trust, Coventry, \\ UK
}

Received 11 November 2013; revised 18 December 2013; accepted 23 December 2013

Copyright (C) 2014 Sian Taylor-Phillips et al. This is an open access article distributed under the Creative Commons Attribution License, which permits unrestricted use, distribution, and reproduction in any medium, provided the original work is properly cited. In accordance of the Creative Commons Attribution License all Copyrights (c) 2014 are reserved for SCIRP and the owner of the intellectual property Sian Taylor-Phillips et al. All Copyright (C) 2014 are guarded by law and by SCIRP as a guardian.

\section{ABSTRACT}

Objective: To test the feasibility of a fully paperless system, termed "paperlite" in a UK breast screening service. To demonstrate in NHS practice, how workload and workflow could be improved by moving to a paperless system and discovering what impact this has upon the complexity within the service. Setting: Warwickshire, Solihull and Coventry Breast Screening Service in the West Midlands of England. Methods: Quality improvement methodologies were employed, including value stream mapping, task analysis and a time-and-motion study. Results: The screening centred screened approximately 50,000 women per year. If they were to implement a paperless system, the administrative workload would decrease. The time saving per batch of screens, which could be achieved by moving to the paperless system ranged from 19 to 56 minutes (mean $=\mathbf{3 6}$ minutes). When calculated by batch the mean time saving per woman screened by moving to the paperless system was 42 seconds. This equates to 583 hours of administrative work per year in a centre screening 50,000 women. Conclusions: The paperless system has many benefits compared to the original system in terms of reductions in waste, time and cost. The simplification and standardisation of the process resulted in fewer tasks and interfaces where errors could occur, hence inadvertently improving patient safety.
The limitation of the work is the heavy reliance on technology, live interfacing with computer databases and software stability is necessary for a paperless system to be used in NHS practice.

\section{KEYWORDS}

Paperless; Breast Screening; Quality Improvement; Process Improvement

\section{INTRODUCTION}

The breast screening programme: The National Health Service Breast Screening Programme (NHSBSP) invites women between the ages of 47 and 73 for regular breast screening (every three years) with the aim of reducing the number of women dying from breast cancer [1]. During 2010-11, 2.44 million women were invited for screening. Of these, 1.88 million (73.4 per cent) were screened and 14,700 cases ( 7.8 per 1000 women screened) of breast cancer were detected [2].

Conventional breast screening (film-screen mammography [FSM]) involves taking four film mammograms of the woman's breasts [3]. The films are developed and displayed on a back-lit multi-viewer for examination for indications of cancer. Digital mammography electronically records and displays the mammograms, and has largely replaced the film screen equipment throughout the UK and worldwide. The implementation of digital image acquisition transfer, storage and display removes the reliance on physically moving $\mathrm{X}$-ray films, and so 
provides an opportunity to redesign workflow and move to paperless systems.

Despite widespread implementation of digital mammography, fully "paperless" systems were not implemented in any UK breast screening centre. The Secretary of State for Health has urged for the "NHS to become a 'paperless' organisation by 2018”, with the aim of “saving billions, improving services and helping meet the challenges of an ageing population" [4], whilst the NHS Commission Board Director is "pushing” for a switchover to digital records by the end of 2015 [5]. Within NHS breast screening services paper-based systems vary in their complexity, but are commonly used in for communication between radiographers, radiologists, administratorsand women. A fully paperless system aims to remove all paper-based process from the system, whereas fully digital mammography uses digitised prior mammograms (mammograms from the previous screening round used by radiologists to assess changes in lesions over time). The availability of digitised prior mammograms removes the need to fetch, hang and replace the woman's prior film mammograms.

The aim of the study is to test the feasibility of a fully paperless system, termed "paperlite" in a UK breast screening service. To demonstrate in NHS practice, how workload and workflow could be improved by moving to a paperless system and discovering what impact this has upon the complexity within the service.

Background to the service: this study was conducted over a five months period within Warwickshire, Solihull and Coventry Breast Screening Service in the West Midlands of England, United Kingdom. The service has three mobile and one static breast screening units where mammograms are taken. An estimated 50,000 women per year are screened with an allocated appointment time of 5.5 minutes. The director of service wished to improve quality and reduce waste in order to meet increasing screening demands with less available resources. Introducing the "paperlite" system was selected as a strategy for improvement. Paperless systems have had reported success in other healthcare organizations [6-11].

\section{METHOD}

\subsection{The Project Team}

An improvement project team was established within the breast screening service. Staff were selected from a range of clinical, managerial and administrative roles within the service. There were no formalised roles or allocated project champions within the team. An additional member of staff was employed for four months to assist with the project and data collection. They took part in workshops throughout the project to bring the team together to and share ideas. The team was assisted by an external researcher who provided support at workshops, board meetings and feasibility sessions.

\subsection{Process Mapping}

During the early workshops the team determined that the greatest opportunity for improvement lay in the administrative process between booking in women for appointments and referral for further investigations. This was perceived as a crucial aspect of the service that could add value to patients (women). A process map of this was created through iterative team discussions and feedback. The sessions were led by the deputy superintendent radiographer who created the first draft of the process map. The project team took part in an iterative process of improving the map, until all members were satisfied that the current process was accurately replicated. The map would be used as a baseline so that future improvements could be highlighted.

\subsection{Task Analysis}

The process steps identified in the process map were categorized during team discussions in a task analysis exercise [12]. Tasks were considered to be Value-added (VA) when they were defined as valuable to the patient and the service. Non-value added (NVA) but business essential tasks were those that were required to keep the service functioning. Waste tasks were those that did not add value to the patient or contribute to efficient running of the service as displayed in Figure 1. The distinction of "value for patients" was as perceived by the project team, not the patients, who primarily value face-to-face contact with health professionals. Nevertheless, there are many tasks which must be performed in order to deliver a safe and effective service. These are likely to go unrecognised by patients - for example, contact with other clinical departments. For this exercise, these tasks were considered to be VA, since they were of direct benefit to the patient.

\subsection{Stakeholder Involvement}

A larger working group was assembled to create the future state process map detailing the paperlite system. This would act as the alternative workflow, which employs paperless reporting to simplify the process. The future state map was disseminated to key stakeholders in the department and at a breast screening staff meeting, for feedback and refinement. It was adapted until all stakeholders were satisfied that there was not a potential increased risk of errors resulting from the proposed changes. The elements of the current process which would no longer be necessary in moving to the paperlite system were identified, and divided into those which would be apparent simply by moving to fully digital mammography, and those which would only materialise through 


\section{Value added tasks}

Planned, opportunistic and reactive patient contact, recording patient notes, care planning, updating patients notes, discussions with other professionals about patient, phone calls to patients, invitation to future appointments
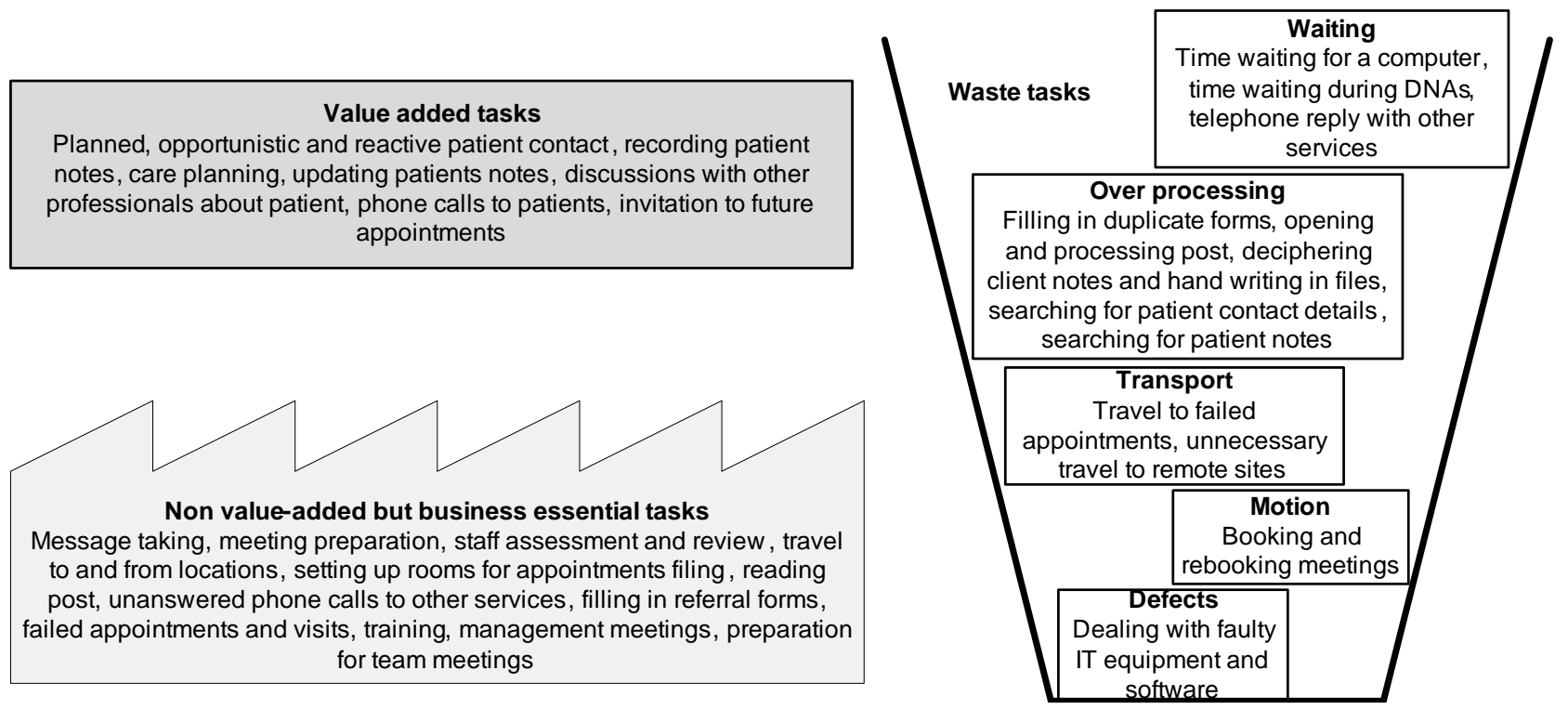

Figure 1. Diagrammatic representation of the tasks identified and categorised in the task analysis exercise.

moving to a paperlite system.

\subsection{Implementation and Time and Motion Study}

Over the course of 30 days, ten batches of mammograms (ten days worth of screening) were followed through the process. Time taken to complete each task was recorded and the quantitative data was collected for a "time-and-motion" analysis. The paperlite system was implemented using a two phased approach. Two laptops were linked via 3G connectivity to each other and to the National Breast Screening computer system using a secure server. This enabled the transfer of information across sites, such as work lists for the day, demographic information and issues reported to the radiographer/assistant practitioner to alert the film reader.

Phase one implementation was for piloting and process improvement, six radiographers followed the paperlite process for five days of screening at one van. Over this period appointment times were extended to $12 \mathrm{~min}-$ utes in preparation for unforeseen issues. Verbal staff feedback was collected and observational notes recorded in a field diary. The aim of phase one was to identify bottle necks and errors in the paperlite system. Smallscale improvement cycles were conducted to ensure the paperlite process did not obstruct the workflow in the breast screening van resulting in increased time taken to perform tasks. During phase two implementation, ten alternative radiographers and assistant practitioners used the new process for an additional five days of screening at the same van incorporating the changes from improvement cycles. This was to assess the effectiveness of the new process map and essentially determine whether the
5.5 minute appointment time could be maintained with the paperlite system. To counter balance errors during this time a contingency administration system operated in the background, this would not form a part of the final implementation. The work was classified as service development and so did not require NHS ethical approvals.

\section{RESULTS}

\subsection{Process Mapping}

Process mapping of the administrative process was carried out by the project team. An example of the map is are presented in Figure 2 (top). It elucidates the large number of processes that are required to carry out this task and the complexity involved. The aim of the improvement team was to redesign the process and eliminate waste steps. Decisions on which steps to remove were made via discussions between clinical, managerial and administrative staff. The redesigned "future state" paperlite process is presented in Figure 2 (bottom). It contains 28 tasks versus the original 42. This represents a $34 \%$ reduction in the number of processes required to carry out one breast cancer screen.

\subsection{Task Analysis}

Table 1 presents the total number of tasks identified and the proportions identified as waste, VA and NVA for the admin, radiography and radiology streams of the current state map. Respectively, $46.6 \%, 25 \%$ and $0 \%$ of the tasks were categorised as waste.

\subsection{Time-and-Motion Study}

Administrative time taken: Paperlite system 


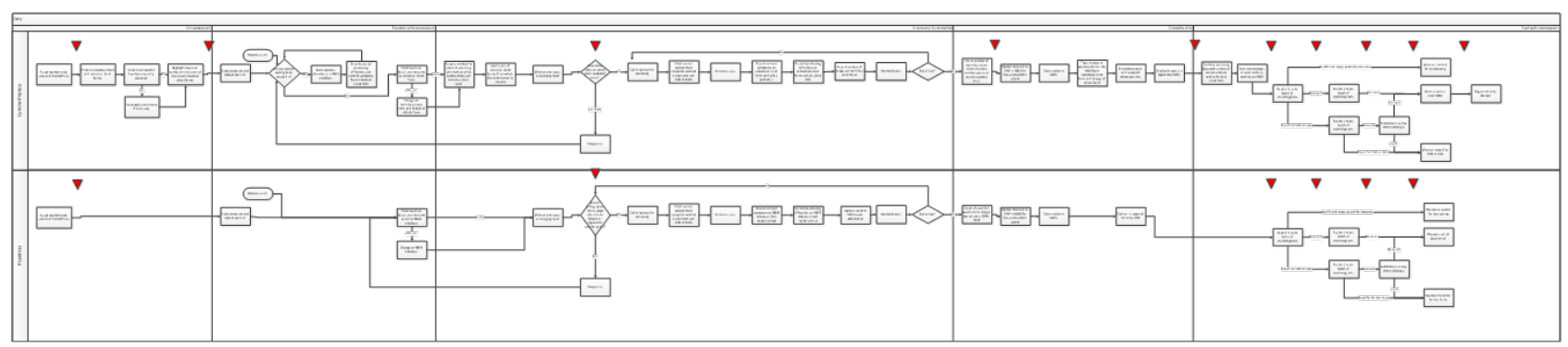

Figure 2. A diagrammatic representation of the current (top) and future state (bottom) breast screening service process maps.

Table 1. Representing the quality and type of tasks identified in the process maps for the office administration element of the breast screening process.

\begin{tabular}{|c|c|c|c|c|}
\hline & \multicolumn{2}{|c|}{ Current state map } & \multicolumn{2}{|c|}{ Future state map } \\
\hline & Number of tasks & Proportion & Number of task & Proportion \\
\hline \multicolumn{5}{|c|}{ Admin task } \\
\hline Value-added task & 4 & $26.6 \%$ & 4 & $50 \%$ \\
\hline Non value-added task & 4 & $26.6 \%$ & 4 & $50 \%$ \\
\hline Waste & $7^{*}$ & $46.6 \%$ & 0 & $0 \%$ \\
\hline \multicolumn{5}{|c|}{ Radiography tasks } \\
\hline Value-added task & 5 & $31.2 \%$ & 5 & $41.6 \%$ \\
\hline Non value-added task & 7 & $43.7 \%$ & 7 & $58.3 \%$ \\
\hline Waste & $4^{* *}$ & $25 \%$ & 0 & $0 \%$ \\
\hline \multicolumn{5}{|c|}{ Radiology tasks } \\
\hline Value-added task & 4 & $100 \%$ & 4 & $100 \%$ \\
\hline Non value-added task & 0 & $0 \%$ & 0 & $0 \%$ \\
\hline Waste & 0 & $0 \%$ & 0 & $0 \%$ \\
\hline
\end{tabular}

* 45 seconds per woman); ${ }^{* *}$ These were not timed due to practical issues in sensitive situation with clients-instead we checked that the 6 minute appointment time could be maintained in the new system, in which the radiographers had some increased administrative duties, but better systems to support them.

The paperlite system reduced the number of activities in the workflow from 42 to 28 . The time saving per batch, which could be achieved by moving to the paperlite system ranged from 19 to 56 minutes (mean $=36$ minutes). This is the time taken to complete all activities that would no longer be necessary, for example typing in and printing out information from the computer database, and matching up computer based records with paper based records. The number of women screened per day, and therefore the number of cases per batch, ranged from 23 to 109 (mean = 52). Overall, when calculated by batch the mean time saving per woman screened by moving to the paperlite system was 42 seconds (range 23 - 69 seconds) see Table 2. This equates to 583 hours of administrative work per year in a centre screening 50,000 women. This is in addition to the time saving through ceasing the necessity to fetch and hang film prior mammograms.

Fully digital system:

The fully digital system with digitised priors reduced the number of steps in the workflow further to 25. The time taken per batch to fetch hang and replace the prior film mammograms varied widely between the batches from 20 minutes to 113 minutes (mean $=59$ minutes). This variation was driven by differing numbers of cases within the batches for which film prior mammograms were available. The mean time saving was 68 seconds per case, (range 42 - 113), equating to an administrative time saving of 944 hours per year when prior mammograms are digital in a centre screening 50,000 women. This is in addition to the saving through moving to a paperlite system. In this sample only $55 \%$ of women had prior mammograms, which in a service offering each women at least seven rounds of screening is likely to be lower than in normal practice. If this is the case the administrative time saving may be an underestimate.

Time taken at the screening van:

Seventy measurements of time taken per woman, on the van reception and in the van X-ray room were taken, from 10 different radiographers. The main source of variability in these measurements originated from the women themselves (for example frailty, discomfort, nervousness) rather than the radiographer. The mean time taken per 
Table 2. Time spent on waste tasks in administration of the breast screening service.

\begin{tabular}{|c|c|c|c|c|c|c|}
\hline \multirow{2}{*}{ Batch } & \multirow{2}{*}{$\begin{array}{l}\text { No. women in } \\
\text { batch }\end{array}$} & \multirow{2}{*}{$\begin{array}{l}\text { No. previous } \\
\text { mammogram }\end{array}$} & \multicolumn{2}{|c|}{$\begin{array}{l}\text { Waste tasks not necessary when } \\
\text { a paperlite system is implemented }\end{array}$} & \multicolumn{2}{|c|}{$\begin{array}{l}\text { Non-value added tasks which will no longer be } \\
\text { necessary when fully digital system is available }\end{array}$} \\
\hline & & & Time spent & $\begin{array}{l}\text { Mean time spent per woman screened } \\
\text { (seconds) }\end{array}$ & Time spent & $\begin{array}{l}\text { Mean time spent per woman screened } \\
\text { (seconds) }\end{array}$ \\
\hline 1 & 23 & 7 & 00:26:38 & 79 & 0:19:49 & 52 \\
\hline 2 & 32 & 17 & 00:19:10 & 36 & $0: 25: 42$ & 48 \\
\hline 3 & 38 & 29 & 00:33:22 & 53 & 0:45:28 & 72 \\
\hline 4 & 56 & 38 & 00:40:42 & 44 & 1:11:36 & 77 \\
\hline 5 & 61 & 39 & $00: 44: 54$ & 44 & 1:15:14 & 74 \\
\hline 6 & 50 & 18 & 00:43:02 & 52 & 0:58:49 & 71 \\
\hline 7 & 55 & 39 & $00: 56: 15$ & 61 & 0:46:00 & 50 \\
\hline 8 & 109 & 43 & $00: 42: 07$ & 23 & 1:48:34 & 60 \\
\hline 9 & 60 & 47 & 00:36:22 & 36 & 1:53:30 & 113 \\
\hline 10 & 36 & 8 & 00:21:17 & 35 & 0:25:11 & 42 \\
\hline $\mathrm{T}$ & 520 & 285 & 06:03:49 & 42 & 9:49:53 & 68 \\
\hline
\end{tabular}

* These waste tasks are all removed when a paperlite reporting system is implemented, tasks include printing from and inputting data to the computer database, and checking different versions are matching. ${ }^{* *}$ These non-value added tasks are no longer necessary when a screening centre has digital prior mammograms, tasks include fetching, hanging, taking down and filing previous film mammograms.

woman in the X-ray room was 5 minutes 12 seconds (95\% CI 2 minutes 33 seconds to 7 minutes 51 seconds). This measure incorporates the changeover time and therefore a 6 minute appointment time can be maintained with the paperlite system. The shortest time taken was 3 minutes 14 seconds and the longest 10 minutes 7 seconds.

In the reception part of the van the mean time taken per woman to book her in and record change of details was 1 minute 4 seconds (range 20 seconds to 2 minutes 30 seconds) dependent on whether the woman's details had changed. On day 8 of the trial the computer in the van reception froze for half an hour. During this time five women attended the van for whom all tasks had to be completed in the X-ray room, time taken in the X-ray room for these women ranged from 3 minutes 21 seconds to 9 minutes 30 seconds (mean 6 minutes).

\section{DISCUSSION}

Improving quality and service processes has been a recent focus of attention for the NHS [13] and the literature available on "quality improvement" in healthcare is continues to grow [14-18]. To date most improvement work has been limited to hospital care, very little has concentrated on primary care and community services such as screening. This study used quality improvement tools such as stakeholder mapping, process mapping and time-and-motion studies to implement a paperless system in a move towards fully digital breast screening.

\subsection{Reduction in Waste and Time}

The initial results from the paperlite system are promising. Seventy measurements of time taken per woman were recorded across 16 radiographers. During the administrate segment of the process, which included "booking in" women and recording personal details, the shortest time taken was 3 minutes 14 seconds and the longest 10 minutes 7 seconds. The mean time taken per woman in the X-ray room was 5 minutes 12 seconds (95\% CI 2 minutes 33 seconds to 7 minutes 51 seconds). The main source of variability in these measurements originated from the women themselves. This result incorporates the changeover time and therefore the 5.5 minute appointment time can be maintained with the paperlite system.

Findings suggest that the paperlite process reduced the number of tasks in the original "current state" process from 42 to 28 . Overall, when calculated by batch the mean time saving per woman screened by moving to the paperlite system was 42 seconds (range 23 - 69 seconds). This equates to 583 hours of administrative work per year in a centre screening 50,000 women. This is in addition to the time saving through ceasing the necessity to fetch and hang film prior mammograms.

\subsection{Improvement in Quality}

The simplification and standardisation of the process resulted in fewer tasks and interfaces where errors could occur, hence inadvertently improving patient safety. It enabled direct entry of information at the time of collection, by the radiographer collecting it rather than an axillary member of staff at a later date. This reduced the number of interfaces between people in the system from 13 to 7, reducing the potential for human error by half. There was significant concern amongst staff before commencing the trial that there may be additional risk because paperlite system reduced the number of checks in 
the process. These concerns were not supported by the results.

Implementation of fully digital mammography is complex and careful planning is required to ensure the existing high quality service by the breast screening centre remains. Collaboration with a wide range of stakeholders across the service during the future state process mapping was essential to its success. This helped to agree specific technical requirements, logistical arrangements and timescales of the paperlite service.

Key staff were involved in the improvement project from the outset. It was acknowledged that the paperlite system would directly impact on staff and their working practices. Staff were encouraged to work in partnership with the project team to ensure their suggestions and issues were addressed. If paperless screening was fully implemented the training requirements of staff would change, policies should be developed to take into account the anticipated changes to the roles and working hours of staff at an early stage.

\subsection{Implementation Challenges}

The immediate results of going paperless are encouraging. However seven critical challenges were identified during process mapping which would have to be overcome for a paperlite system to be implemented and accepted within a breast screening service.

1) Instant savings in administration time at the screening centre must be revealed to justify the change. This is important for initial staff engagement.

2) The process changes at the screening van must not increase the appointment time required per woman above 5.5 minutes

3) The paperlite system must not increase the risk of errors compared to the original paper process. For example mistakes in patient notes.

4) There must be an effective communication system between the operator taking the mammograms and the radiologist reading the mammograms

5) Reliable IT equipment and software is essential to the success of a paperlite system. Fast internet connections and regular back-ups are a necessity, and cannot be overlooked during pre-project planning

6) Contingency plans need to be in place prior to "go-live" these need to be documented to ensure operators are confident in knowing what to do if computer systems fail

7) Information Governance needs to be maintained when using the paperlite processes.

These challenges are being overcome at the study centre, and a paperlite system has been implemented to normal practice for part of the service, with plans to extend to the whole service as the IT connectivity is stabi- lised.

Limitations of the study: the 30-day time frame of the study limits the validity of the findings. It does not reflect any seasonality or media influence that may exists in breast screening services, these may impact upon appointment timing and workload.

This study was limited to a small scale implementation at one breast screening service in the UK. Identification of waste process steps was relatively straightforward; the challenging aspect was how to remove waste and sustain the identified improvements in the future. The complexity of the service meant that a large number of stakeholders had to be involved to implement even the simplest change. It became clear that meaningful change would require input from management across many departments.

Future research: fully paperless reporting should be piloted at a wider scale at the earliest opportunity. A full implementation study would aim to extend across more than one screening service in the region. Future research would investigate the implementation of fully digital screening across other centres over a fixed period of time.

\section{CONCLUSION}

Fully paperless breast screening saves time and should be implemented as soon as breast screening centres have previous digital mammograms available. The priority challenges when "going paperless" are to guarantee stable and efficient information technology connectivity between mobile breast screening vans and hospital sites and sustainable systems of communication between the radiographers performing the scans and the radiologists who read them. Over coming, these challenges will ensure a safe, effective, patient-centred, timely, efficient and equitable breast screening service.

\section{ACKNOWLEDGEMENTS}

The authors would like to thank the staff at the Warwickshire, Solihull and Coventry Breast Screening Service for their help and support during the improvement project. This study was funded by the University Hospital Coventry and Warwickshire trust fund. Ethical approval was not required for the study as it was categorised as a service improvement project. The authors have no conflicts of interest to report.

\section{REFERENCES}

[1] NHS Breast Screening Programme (2012) NHS Breast Screening Programme 2012 Annual Review. http://www.cancerscreening.nhs.uk/breastscreen/publicati ons/2012review.html

[2] NHS Information Centre (2012) Breast Screening Pro- 
gramme, England 2010-11. The Health and Social Care Information Centre, Screening and Immunisations team. https://catalogue.ic.nhs.uk/publications/screening/breast/b res-scre-prog-eng-2010-11/bres-scre-prog-eng-2010-11-re p.pdf

[3] ONHS Quality Improvement Scotland (2008) Health Technology Assessment 10 determining the most clinically and cost-effective way of implementing digital mammography services for breast screening in NHS Scotland. www.nhshealthquality.org

[4] Cross, M. (2013) Experts question size of savings from NHS going “paperless”. BMJ, 346, f438. http://dx.doi.org/10.1136/bmj.f438

[5] Limb, M. (2012) NHS will be paperless by 2015, says commissioning board. BMJ, 345, e6888. http://dx.doi.org/10.1136/bmj.e6888

[6] Purves, I.N. (1996) The paperless general practice. BMJ, 4, 1112-1113. http://dx.doi.org/10.1136/bmj.312.7039.1112

[7] Waring, N. (2000) To what extend are practices "paperless" and what are the constraints to them becoming so? BMJ, 50, 46-47.

[8] Carr-Bains, S. and de Lusignan, S. (2003) Moving to paperlessness: A case study from a large general practice. Informatics in Primary Care, 11, 157-163.

[9] Hippisley-Cox, J., Pringle, M., Cater, R., et al. (2003) The electronic patient record in primary care-regression or progression? A cross sectional study. BMJ, 326,1439. http://dx.doi.org/10.1136/bmj.326.7404.1439

[10] Harvey, J. (2008) End of the paper trail: Moving towards a paperless ward round. The Psychiatrist, 32, 67-68. http://dx.doi.org/10.1192/pb.bp.106.014225
[11] Allan, R. (2010) Paperless NHS hospital medical records. Clinical Medicine, 10, 315. http://dx.doi.org/10.7861/clinmedicine.10-4-315

[12] Grove, A.L., Meredith, J.O., MacIntrye, M., Angelis, J. and Neailey, K. (2010) Lean implementation in primary care health visiting services in National Health Service UK. Quality \& Safety in Health Care, 19, 1-5. http://dx.doi.org/10.1136/qshc.2009.039719

[13] Department of Health (2013) Quality in the new health system. Maintaining and improving quality from April 2013. A draft report from the National Quality Board. https://www.wp.dh.gov.uk/publications/files/2012/08/nqb -quality-draft.pdf

[14] Fillingham, D. (2007) Can lean save lives? Leadership in Health Services, 20, 231-241.

[15] Esain, A., Williams, S. and Massey, L. (2008) Combining planned and emergent change in a healthcare lean transformation. Public Money \& Management, 28, 21-26.

[16] Wilson, G. (2009) Implementation of releasing time to care-The productive ward. Journal of Nursing Management, 17, 647-654. http://dx.doi.org/10.1111/j.1365-2834.2009.01026.x

[17] Roueche, A. and Hewitt, J. (2012) "Wading through treacle": Quality improvement lessons from the frontline. BMJ Quality and Safety, 21, 179-183. http://dx.doi.org/10.1136/bmjqs-2011-000545

[18] Kaminski, G.M., Britto, M.T., Schoettker, P.J., Farber, S.L., Muething, S. and Kotagal, U.R. (2012) Developing capable quality improvement leaders. BMJ Quality and Safety, 21, 903-911. http://dx.doi.org/10.1136/bmjqs-2012-000890 